\title{
Maltrato físico e inteligencia emocional en niños y niñas de una institución educativa, Huancavelica
}

\author{
Physical abuse and emotional intelligence in children from an educational institution, \\ Huancavelica
}

Huamán Albites, Margarita Isabel 1,a, Nieva-Villegas Lilia María 2,b , Gómez Gonzales Walter Edgar,c

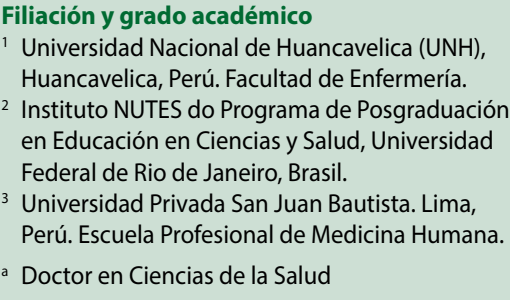

NVLM: Concepción de la investigación, formulación del proyecto, análisis de datos, redacción de artículo.

GGWE: Análisis de datos, asesoría estadística, redacción del artículo.

Fuentes de financiamiento

Universidad Nacional de Huancavelica.

Conflictos de interés

Ninguno.

Recibido: 30-10-2020

Arbitrado por pares

Aceptado: 01-12-2020

Citar como

Huamán M, Nieva-Villegas L, Gómez-Gonzales W. Maltrato físico e inteligencia emocional en niños y niñas de una institución educativa, Huancavelica. Rev Peru Cienc Salud. 2020; 2(4): 257-65. doi: https://doi.org/10.37711/rpcs.2020.2.4.227

\section{RESUMEN}

Objetivo. Determinar la relación entre el maltrato físico y la inteligencia emocional en niños y niñas de una institución Educativa de Huancavelica. Métodos. Investigación de tipo observacional, correlacional, transversal; se tomó una muestra de 48 niños(as), entre 10 y 13 años; se utilizaron la técnica psicométrica, la entrevista semiestructurada y la escala de Likert. Resultados. El tipo de maltrato físico se evidencian con el uso del jalón de orejas, golpes con correa, golpes con la mano o cachetadas, patadas, golpes con chicote, con palo o con soga, pellizcos y empujones. La madre apareció como la principal agresora, seguido de padres y hermanos. El maltrato fue severo en un $24 \%$, moderado en un $21 \%$ y leve en un $13 \%$. Las niñas fueron las menos maltratadas en comparación con los niños. No hubo diferencias significativas por nivel de maltrato respecto al manejo del estrés, de una adecuada a atípica capacidad; respecto al estado de ánimo, las víctimas de maltrato mostraron una baja a deficiente capacidad, siendo en primer lugar el moderado, seguido del severo y, por último, el leve. Conclusión. Existe relación negativa baja o no significativa ( $r s=-0,156, p>0,05$ ) entre el maltrato físico y la inteligencia emocional, al encontrarse una inteligencia emocional de adecuada a alta, independiente del nivel de maltrato físico.

Palabras clave: inteligencia emocional; maltrato físico-psicológico (Fuente: DeCSBIREME).

\section{ABSTRACT}

Objective. To determine the relationship between physical abuse and emotional intelligence in children from an educational institution in Huancavelica. Methods. Observational, correlational, cross-sectional research; a sample of 48 children, between 10 and 13 years old, was taken; the psychometric technique, the semi-structured interview and the Likert scale were used. Results. The type of physical abuse is evidenced by the use of earpulling, hitting with a belt, hitting with the hand or slapping, kicking, hitting with a chicote, stick or rope, pinching and pushing. The mother appeared as the main aggressor, followed by parents and siblings. The abuse was severe by $24 \%$, moderate by $21 \%$ and mild by $13 \%$. Girls were the least abused compared to boys. There were no differences by level of abuse, regarding stress management, from adequate to atypical capacity; with regard to the state of mind, the victims of abuse showed a low to poor capacity, being first the moderate one, followed by the severe one and, finally, the mild one. Conclusion. There is a low or non-significant negative relationship ( $\mathrm{rs}=$ $-0.156, p>0.05$ ) between physical abuse and emotional intelligence, as an adequate to high emotional intelligence is found, regardless of the level of physical abuse.

Keywords: emotional intelligence; physical-psychological abuse (Source: MeSH NLM). 


\section{INTRODUCCIÓN}

El maltrato infantil es un fenómeno que es intrínseco a la aparición de la civilización siendo tan antiguo como la humanidad. Su abordaje como un problema grave inicia con el síndrome del niño golpeado descrito por primera vez en 1868 por Ambrosie Tardieu, luego de realizar autopsias de 32 niños golpeados y quemados. Posteriormente, en 1946, Caffey describió la presencia de hematomas subdurales asociados con alteraciones radiológicas de los huesos largos en los niños, creándose la expresión del síndrome del niño golpeado, por Henry Kempe y Silverman, en $1962{ }^{(1)}$. Este problema se registra, por lo general, en los espacios más íntimos de la convivencia familiar, en donde los padres, la fuente más importante de afecto para los niños(as), son quienes ejercen alguna forma de violencia física como una medida de disciplina que incluye insultos y desprecios, entre otros, haciendo que los niños(as) se sientan impotentes y desanimados para enfrentar estas experiencias ${ }^{(2)}$.

En el Perú existen señales de la existencia de este fenómeno en lo reportado por el Ministerio de la Mujer y Poblaciones Vulnerables, a través del Programa Nacional contra la Violencia Familiar y Sexual. Los casos atendidos en los Centros de Emergencia Mujer, a nivel nacional en el año 2016 reportaron 9270 denuncias de maltrato psicológico; 9,3\% en el grupo de 0 a 5 años, el 43,7 \% entre los 6 y 11 años y el 36,96 \% entre 12 y 17 años. Respecto al maltrato físico, de un total de 7867 casos, el 21,9\% se registró entre 0 y 5 años, el 40,8 \% entre 6 y 11 años y el 37,3\% entre los 12 y 17 años. En el año 2017 se observó un incremento de los casos de maltrato psicológico a 13830 , encontrando un $19,9 \%$ de 0 a 5 años, el 44,5 \% entre 6 y 11 años y el 35,6 \% entre 12 y 17 años. El maltrato físico también evidencia un incremento a 10142 casos, siendo el $21,4 \%$ de 0 a 5 años, el 40,7 \% entre 6 y 11 años y de 37,9\% entre 12 y 17 años.

Finalmente, para el año 2018, los casos de maltrato psicológico se hacen más evidentes, elevándose a 18911 casos, el 21,0 \% de 0 a 5 años, el 43,7 \% entre 6 y 11 años y el 35,2 \% entre 12 y 17 años; en tanto que el maltrato físico se elevó a 13752 casos, el 19,9\% de 0 a 5 años, el 39,2 \% entre 6 y 11 años y el 40,8 \% entre 12 y 17 años. Como puede observarse, los casos van en aumento cada año, siendo el grupo más afectado de esta población los de 6 a 11 años ${ }^{(3)}$. Encuesta Nacional sobre Relaciones Sociales (ENARES 2015), revela que el $42,9 \%$ de los padres de familia está de acuerdo con que a veces es necesario gritar a los niños y adolescentes para que entiendan lo que se les dice. Otro $36,1 \%$ indica que se debe castigar físicamente al niño o adolescente si se mete en problemas, miente con frecuencia o coge algo que no le pertenece ${ }^{(4)}$.

Además, estudios a nivel nacional como el de Trigoso, refrendan la existencia de este fenómeno, al reportar en una muestra de 63 niños, 50,8 \% víctimas de maltrato físico, correspondiéndose un $38,1 \%{ }^{(5)}$ con el maltrato físico severo, un $11,12 \%{ }^{(6)}$ con maltrato físico moderado y un $1,6 \%{ }^{(1)}$ con el maltrato físico leve ${ }^{(7)}$. Así mismo Salcedo, de una muestra de 183 niños y niñas. Asimismo, en una muestra de 9 a 12 años de edad, Salcedo identificó la presencia del maltrato físico en el 53,6 \% en un nivel medio, el 33,3 \% en un nivel bajo y el $13,1 \%$ en un nivel alto. El maltrato infantil estuvo presente en los niños, con un $68,9 \%$ en un nivel medio y el $13,3 \%$ en un nivel alto; en tanto que en las niñas, el 61,3\% reflejó un nivel medio y el $15,1 \%$ un nivel alto ${ }^{(8)}$.

La Región de Huancavelica no es ajena a este fenómeno, evidenció en estudios de Huaman, con una muestra de 36 niños(as), entre de 10 a 12 años, distribuidos equitativamente en un grupo experimental y un grupo control encontró los siguientes resultados. Respecto del maltrato físico, encontró su existencia al en la categoría de moderado, en un $66,7 \%$ en el grupo experimental y un $77,8 \%$ en el grupo control, infringido principalmente por la madre, seguido de la madre-padre y hermanos. Respecto a las formas, en las niñas el 38,7\% era mediante jalón de orejas, el 36,1 \% jalón de cabellos, el 27,7 \% empujones y pellizcos, el 22,2 \% correazos, el 11,1\% patadas, el 8,3\% cachetadas, chicotazos y cocachos y el 2,7 \% palazos. En niños, el 38,8 \% correazos, el 30,5 \% jalones de cabello y de orejas, el $22,2 \%$ pellizcos y chicotazos, el $11,1 \%$ patadas y cocachos y el 5,5\% cachetadas, empujones, palazos y el que le hayan echado agua y golpearon ${ }^{(9)}$.

Del mismo modo, en los estudios de Carhuachuco, en un total de 270 estudiantes, el 28,5 \% fueron víctimas de maltrato físico; con respecto a las formas de maltrato físico, el 91,85\% fue golpeado con objetos, el 34,81\% fue abofeteado, el 32,59\% recibió patadados, el 25,19\% fue pegado con el puño, el 23,70 \% fue pellizcado, el $15,93 \%$ fue herido con arma, el 14,07 \% fue mordido, el $11,11 \%$ fue tratado de ser estrangulado y el 5,19\% fue quemado ${ }^{(10)}$. Igualmente, Belito y Gaspar, reportaron un $50,8 \%$ niños víctimas de maltrato físico, un $42,4 \%$ de maltrato psicológico y un $6,8 \%$ de abuso sexual, de un total de 264 estudiantes ${ }^{(11)}$. Y Castrejón, quien encontró víctimas de maltrato físico, con un $53,92 \%$ en niños y un $46,08 \%$ en niñas, siendo las formas de maltrato de un $50,0 \%$ de bofetadas, un $26,47 \%$ de látigos, un $6,86 \%$ de patadas y un $16,47 \%$ de otros tipos ${ }^{(12)}$. 
De lo expuesto, el maltrato infantil y, específicamente, el maltrato físico, se constituye en un factor de riesgo que afecta la salud mental y afectiva de la víctima; fenómeno que se constituye en una barrera que impide el desarrollo psico-social evolutivo normal de los niños (as). En este contexto se tuvo como objetivo determinar la relación entre el maltrato físico y la inteligencia emocional en niños y niñas de la Institución Educativa $N^{\circ} 36005$ del Distrito de Ascensión, en Huancavelica. Teniendo como hipótesis: existe relación entre el maltrato físico y la inteligencia emocional en niños y niñas de la Institución Educativa N³6005 del distrito de Ascensión, Huancavelica.

\section{METODOLOGÍA}

\section{Tipo de estudio}

El trabajo de investigación se corresponde con el tipo observacional, dado que las características del fenómeno son susceptibles de ser observadas en un tiempo y contexto determinando. De nivel correlacional, porque se midió el nivel de relación entre las variables "maltrato físico" e "inteligencia emocional", se estableció la relación entre variables sin exigir sentido de causalidad o pretender relaciones causales. Además, de acuerdo a Uculmana y Lanchipa, es correlacional por que mide la relación positiva o negativa entre dos o más conceptos o variables y, después, analiza la relación entre ellas ${ }^{(13)}$.

\section{Área del estudio}

El estudio se desarrolló en la Institución Educativa № 36 005, de nivel primario, perteneciente al Ministerio de Educación y que es, por lo tanto, de condición pública, ubicada en el distrito de Ascensión, dentro de la jurisdicción de la Dirección Regional de Educación y la UGEL Huancavelica, Perú. Esta institución alberga niños y niñas entre 6 y 12 años de edad. La investigación se realizó en el año 2019.

\section{Diseño del estudio}

Se trató de un diseño no experimental, porque careció de la manipulación intencional de las variables y solo se analizaron y estudiaron los hechos y fenómenos de la realidad después de su ocurrencia. Asimismo, fue Transversal porque permitió realizar la medición del fenómeno en una realidad y en un momento determinado. Finalmente fue también correlacional, porque tuvo como propósito evaluar la relación que existe entre las dos variables de estudio, como son el maltrato físico y la inteligencia emocional.

\section{Población y muestra}

La población estuvo constituida por 83 niñas y niños del $5^{\circ}$ y $6^{\circ}$ grado de estudios, que asistieron a la
Institución Educativa № 36005 del Distrito de AscensiónHuancavelica. Fue seleccionada mediante un método no probabilístico e intencional y estuvo constituida por niñas (os) entre 10 y 13 años, víctimas de maltrato físico, haciendo un total de 48. Criterios de inclusión: Estudiantes del $5^{\circ}$ y $6^{\circ}$ grado de estudios que asisten a la Institución Educativa № 36005 del Distrito de Ascensión, Huancavelica; estudiantes comprendidos entre 10 y 13 años de edad; estudiantes en cuyos instrumentos se identificaron 1 o más ítems marcados, que señalan haber sido víctimas de maltrato físico; estudiantes que viven con papa, mama, hermanos y otros; estudiantes que viven con mama, hermanos y otros; estudiantes que viven con hermanos; estudiantes que viven con abuelas(os). Criterios de exclusión: estudiantes que viven en la Aldea Infantil San Francisco de Asís, Huancavelica.

\section{Instrumentos de relección de datos}

Para la identificación de las víctimas de maltrato físico se empleó la técnica de la entrevista y su instrumento: la guía de entrevista estructurada. Para la variable inteligencia emocional se empleó la técnica psicométrica como instrumento la escala de Likert, Evaluación Emocional BarOn ICE: NA - versión completa ${ }^{(1)}$. Adaptada por Nelly Ugarriza Chávez y Liz Pajares del Águila, que consta de 60 items ${ }^{(14)}$.

\section{Procedimientos de la recolección de datos}

Previa coordinación con las autoridades de la Institución Educativa, se realizó la validez y confiabilidad del instrumento de recolección de datos y, a la vez, se capacitó a los encuestadores, quienes tendrían la responsabilidad de aplicar los instrumentos. Posteriormente, se aplicó el instrumento para medir el maltrato físico en un ambiente separado, cuidando la privacidad de cada alumno $y$, posteriormente, se realizó el tamizaje a fin de identificar solo a las víctimas de maltrato. Finalmente, se aplicó el inventario emocional a las víctimas de maltrato cuidando, de igual forma, la individualidad.

Los datos fueron procesados y analizados en el PASW Statistic Vers. 20,0, Stata 6,0 para el modelo de ajuste y la Statistica 8,0 para la presentación de tablas y gráficos. Los resultados se presentan en la primera sección a través de la estadística descriptiva, abordando los datos generales, la descripción en base a los objetivos específicos (caracterización de cada variable de estudio, análisis descriptivo de relación entre variables y sus componentes) y el objetivo general. En la segunda parte se detalla el proceso de contraste de hipótesis, que emplea la prueba de rangos de Spearman; considerando a ambas variables de estudio en un nivel de medición ordinal. 


\section{Aspectos éticos}

Se han recogido los consentimientos informados de los padres de los estudiantes encuestados, previa explicación de los procedimientos.

\section{RESULTADOS}

Se observa que la muestra de estudio estuvo conformada principalmente por estudiantes de quinto (49\%) y sexto grado (51\%) de primaria, de 10 a 13 años de edad, con mayor proporción de niños (54\%) en comparación a las niñas (46\%). El $74 \%$ de los niños provenía de hogares funcionales sin hermanos (11\%) y con hermanos (50\%), hogares extendidos con otros parientes (13\%) y hogares disfuncionales (24\%) con uno de los padres (21\%), con hermanos ( $1 \%$ ) y con tíos (2\%). El $2 \%$ de encuestados vivía en un albergue.

De los 83 estudiantes encuestados, el tipo de maltrato físico que se presentó "a veces" fue, principalmente. jalón de orejas (34\%), los golpes con correa (29\%), los golpes con la mano o cachetadas (20\%), las patadas y golpes con chicote (13\% cada uno), los pellizcos (11\%), los empujones y golpes con palo ( $10 \%$ cada uno) y los golpes con soga (6\%). Así mismo, el tipo de maltrato que se ejerció "con la frecuencia de siempre" fue: pellizcos (2\%), jalón de orejas, golpes con palo, quemaduras e intento de estrangulamiento ( $1 \%$ cada uno).

Se observa que, 32 madres de familia ejercieron algún grado de maltrato sobre los estudiantes, al igual que 25 padres de familia, 19 hermanos mayores, 2 tíos, 1 cuñado, 1 abuelo y 1 padrastro. Así mismo, el grado de maltrato físico que ejerció el padre fue principalmente moderado (56\%), severo (28\%) y, por último, leve

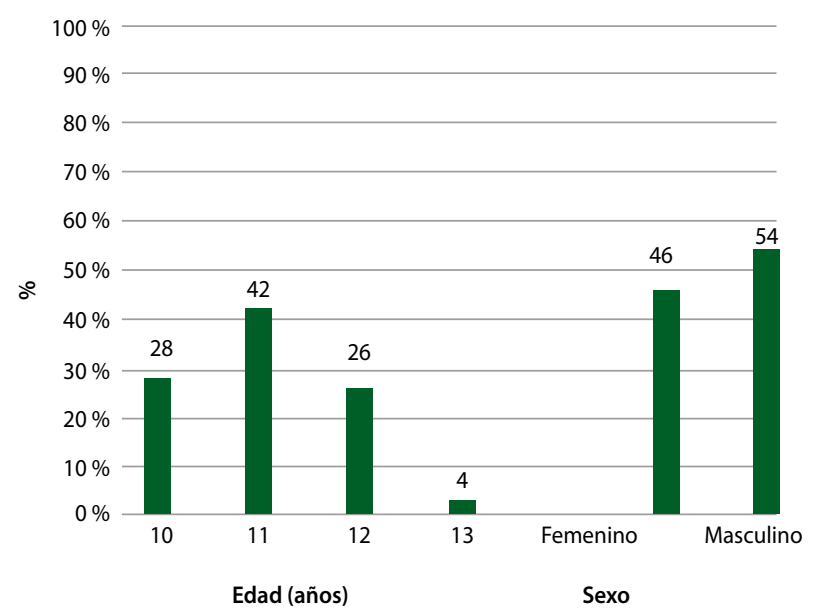

Figura 1. Características generales de los encuestados (edad, sexo)

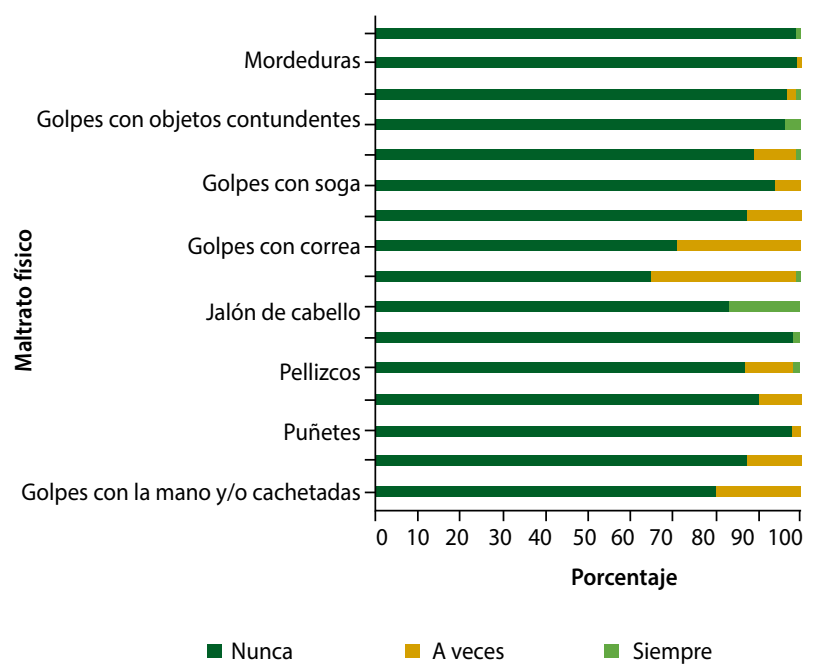

Figura 2. Características del maltrato infantil que sufren los encuestados

(16\%); Por otra parte, el grado de maltrato que ejerció la madre fue mayormente, leve (38\%), pero con pocas diferencias con respecto al maltrato moderado y severo (31\% respectivamente). Los hermanos mayores ejercieron principalmente un maltrato moderado a severo $(37 \%$ cada uno), seguido de leve ( $26 \%)$. Los tíos y padrastro sólo ejercieron maltrato severo. El cuñado y los abuelos sólo ejercieron un maltrato de grado leve.

Tras el maltrato físico, 38 niños sintieron tristeza, 8 sintieron dolor, 7 se sintieron enojados, 4 experimentaron miedo, 3 se sintieron deprimidos y 2 niños sintieron intranquilidad o arrepentimiento. Además, de los niños que sintieron miedo, el $50 \%$ sufrió maltrato físico severo, el $25 \%$ maltrato leve y moderado, respectivamente. De los que sintieron tristeza, el $45 \%$ experimentó maltrato severo, el $31 \%$ moderado y el $24 \%$ leve. Del total que

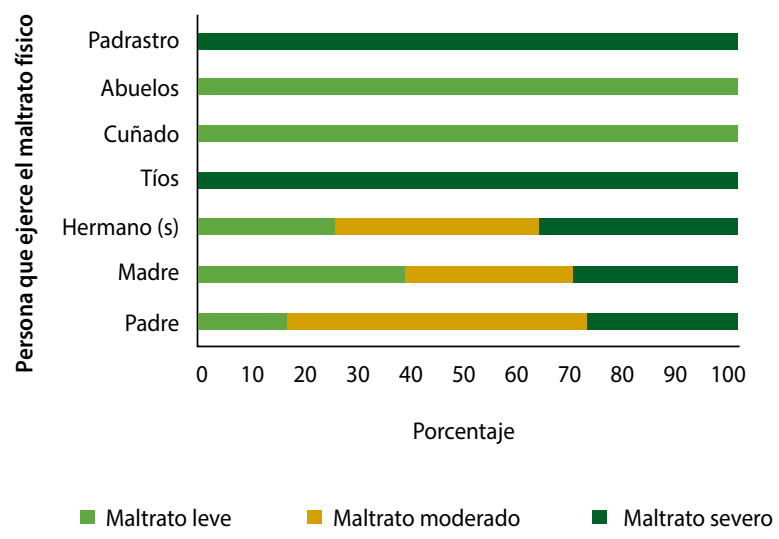

Figura 3. Personas que ejercen el maltrato infantil 


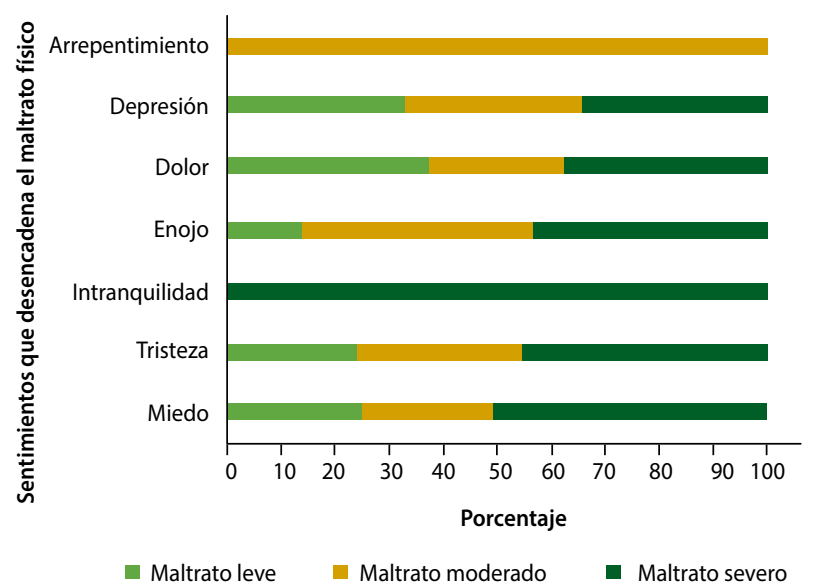

Figura 4. Reacciones experimentadas después del maltrato infantil

sintió enojo, el 43 \% sufrió maltrato moderado y severo, respectivamente, y el $14 \%$ maltrato leve. Los niños que sintieron dolor experimentaron maltrato leve o severo en $37,5 \%$ respectivamente, $y$, en $25 \%$ maltrato moderado. Del total de niños que se sintieron deprimidos, el 33,33\% experimentó maltrato leve, moderado o severo, respectivamente. Por último, el niño que se sintió arrepentido sufrió maltrato moderado.

Se evidencia que la impresión positiva fue de 105,51 $\pm 11,98$, con una mediana de 105,13; y una distribución asimétrica negativa $(-0,33)$ y leptocúrtica $(1,43)$. Esto indica que la tendencia positiva de respuestas fue adecuada. El índice de consistencia es de 7,31 $\pm 3,24$, con una mediana de $7, y$ una distribución asimétrica positiva $(0,59)$ y leptocúrtica $(0,36)$. Como el índice de consistencia fue menor que 10 , las respuestas de los estudiantes fueron aceptables. En general, la inteligencia emocional tuvo un promedio de $107,38 \pm 9,21$, con una mediana de 108,37 (adecuada) y una distribución asimétrica negativa $(-0,08)$ y platicúrtica $(-0,57)$. En la dimensión intrapersonal, el promedio fue de 114,18 $\pm 13,91$, con una mediana de 114,10 (alta) y una distribución asimétrica

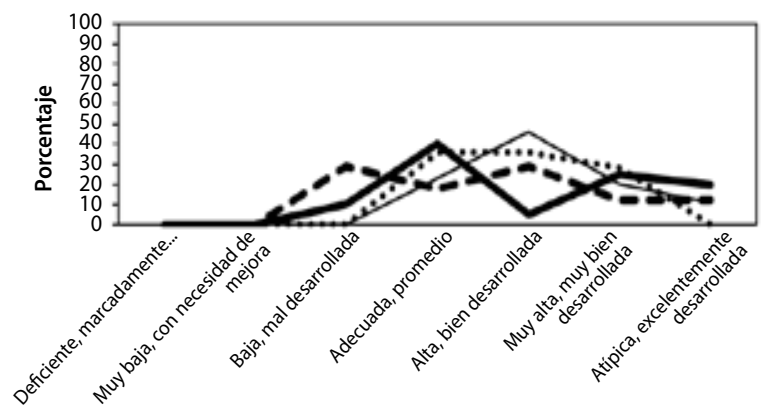

Capacidad emocional y social intrapersonal

Nivel de maltrato

- Ninguno …..... Leve --- Moderado Severo

Figura 6. Capacidad emocional y social interpersonal

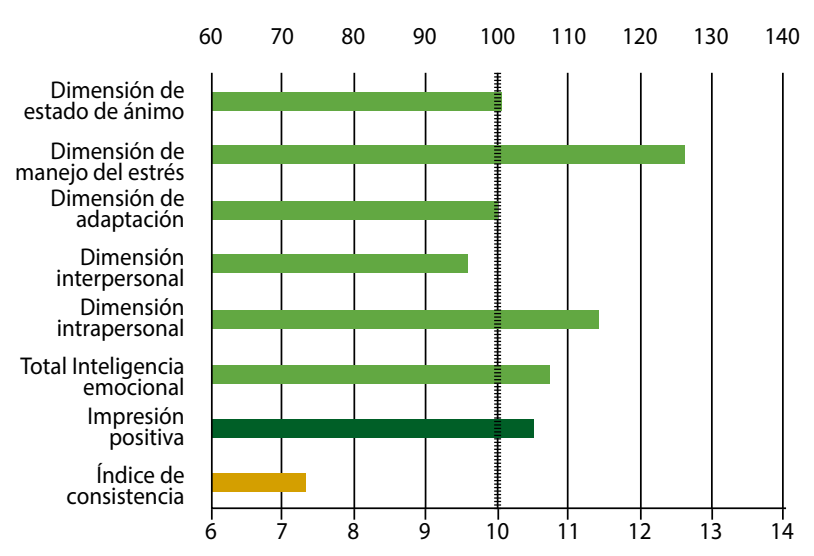

Figura 5. Inteligencia emocional según dimensiones

negativa $(-0,18)$ y normocúrtica $(-0,05)$. En la dimensión de adaptación, el promedio fue de $99,85 \pm 16,31$, con una mediana de 101,02 (adecuada) y una distribución asimétrica negativa $(-0,29)$ y platicúrtica $(-0,58)$. En la dimensión de estado de ánimo, el promedio fue de 100,78 $\pm 15,34$, con una mediana de 103,45 (adecuada) y una distribución asimétrica negativa $(-0,70)$ y normocúrtica $(-0,02)$.

Puede observarse que el $32 \%$ de los niños que no sufrieron maltrato físico tienen una capacidad emocional y social de adaptación entre baja y deficiente; esta misma tendencia se observa en el $18 \%$ de estudiantes que sufrieron maltrato físico leve. En cuanto al maltrato físico moderado y severo la tendencia de capacidad, entre baja y deficiente, es del $30 \%$ y $35 \%$, respectivamente. Esto implica que no hay diferencias importantes entre los niveles de capacidad emocional de adaptación y los niveles de maltrato físico. Los niños que no experimentaron maltrato físico, tuvieron entre adecuada y atípica capacidad emocional y social de manejo del estrés, al igual que los niños que experimentaron algún grado de maltrato físico. Esto indica que no existen diferencias importantes entre

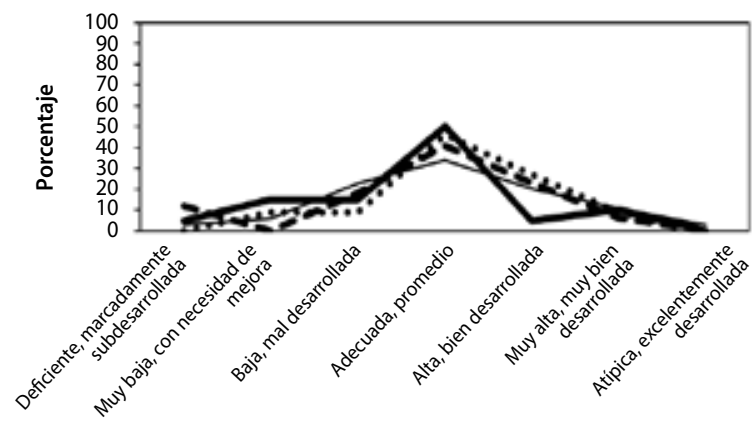

Capacidad emocional y social de adaptación

Nivel de maltrato

- Ninguno ......... Leve ----- Moderado - Severo

Figura 7. Capacidad emocional y social de adaptación 


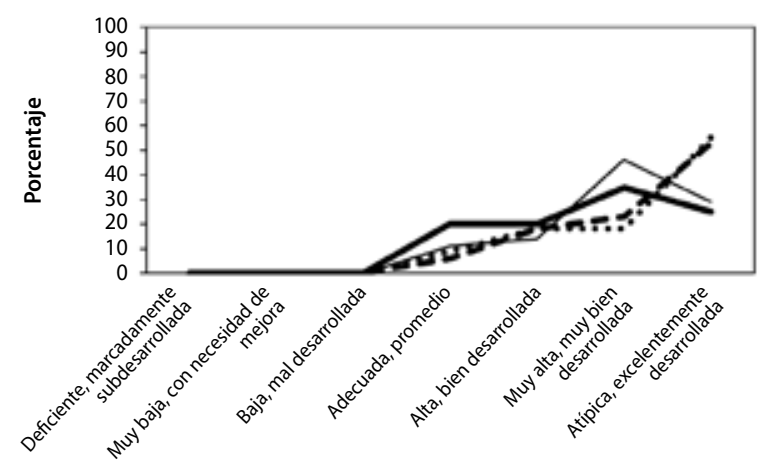

Capacidad emocional y social de manejo del estrés

Nivel de maltrato

- Ninguno ......... Leve ---- Moderado - Severo

Figura 8. Personas que ejercen el maltrato infantil.

la capacidad de manejo estrés en base al nivel de maltrato físico.

Se observa una tendencia entre adecuada y alta inteligencia emocional, en el grupo de niñas y niños que no sufrieron ningún tipo de maltrato físico y en los que experimentaron algún nivel de maltrato

Como puede observarse en la figura, con respecto a la hipótesis general, el coeficiente de correlación de Spearman entre maltrato físico e inteligencia emocional es de $-0,156$, mayor que -0,216; por lo tanto, no se puede rechazar la hipótesis nula, con una significación de 0,160 , mayor que 0,05 ( $p>0,05$ ). Esto implica que no hay suficiente evidencia de relación significativa entre el maltrato físico y la inteligencia emocional en los niños y niñas de la I.E. N³6 005 del distrito de Ascensión, Huancavelica.

\section{DISCUSIÓN}

Gallegos, define el maltrato físico como cualquier lesión corporal que un padre, cuidador o cualquier otro miembro de la familia cause o pudiera causar lesiones o daños físicos a un niño, estén relacionados o no con la disciplina ${ }^{(7,8)}$, considerado como indicadores visibles; magulladuras o moretones, quemaduras, fracturas, torceduras o dislocaciones, heridas o raspadura, señales de mordeduras humanas, cortes o pinchazos, etc. El maltrato físico encontrados en nuestro estudio, correspondió en mayor porcentaje con el severo, seguido del moderado y en un menor porcentaje con leve. En la categoría de severo (golpes con correa, patadas, puñetes, golpes con chicote, objetos, soga y palo, mordidas, intentos de estrangulamiento, quemaduras) que representó el mayor porcentaje, seguido del moderado (jalón de orejas, jalón de cabellos, cachetadas y cocachos), las víctimas fueron los niños, en tanto que en la categoría de leve (empujones y pellizcos), las niñas fueron más maltratadas que los niños. Según el análisis por categoría de maltrato físico, los resultados fueron similares en la categoría de severo, pero diferentes en las categorías de moderado y leve respecto al estudio de Peña ${ }^{(15)}$ en un $36,4 \%$ víctimas de maltrato físico en un nivel alto, $28,4 \%$ en un nivel moderado y $35,2 \%$ en un nivel bajo. Por su parte, Bautista ${ }^{(16)}$ reportó un $50 \%$ de víctimas de maltrato físico alto, un $45 \%$ bajo y un $5 \%$ regular. Unos resultados diferentes a los hallados por Saucedo ${ }^{(17)}$, al encontrar un $62,90 \%$ de maltrato físico de baja intensidad o leve, $33,87 \%$ intensidad media o moderado y 3,23\% de alta intensidad o fuerte, o los de Belito y Gaspar (19) al reportar un $47,8 \%$ de víctimas de maltrato físico moderado, $38,1 \%$ leve y $14,2 \%$ severo.

En términos generales, la presencia de maltrato físico reportado por Castrejón ${ }^{(22)}$, Huaman ${ }^{(18)}$, Bautista ${ }^{(16)} y$ Peña ${ }^{(15)}$, evidencian la presencia de este flagelo en un mayor porcentaje en niños respecto de las niñas, corroborando los resultados encontrados en nuestro estudio; lo que nos permite concluir que los niños son los que están más expuestos a este tipo de maltrato. De otro y respecto a la variable edad en las víctimas de maltrato físico, en la categoría de leve, el maltrato más frecuente se dio entre los 10 y 12 años; el moderado principalmente a los 13 años y de manera menos frecuente a los 12 años; y el severo se presentó en los 10 años. Cuanto mayor fue la edad, menor fue el nivel de maltrato físico.

Los niños(as) que no sufrieron ningún maltrato físico tuvieron entre adecuada y atípica capacidad emocional y social intrapersonal; los que experimentaron maltrato leve tuvieron una capacidad entre adecuada y muy alta;

Tabla 1. Prueba de correlación de rangos de Spearman para la hipótesis general

\begin{tabular}{cccccc}
\hline Variable $\mathbf{X}$ & Variable $\mathbf{Y}$ & $\mathbf{N}$ & Valor & $\begin{array}{c}\text { Error estandarizado } \\
\text { asintóticoa }\end{array}$ & $\begin{array}{c}\text { T aproximadab } \\
\text { aproximadac }\end{array}$ \\
\hline Maltrato físico & Inteligencia emocional & 83 & $-0,156$ & 0,108 & $-1,419$ \\
\hline
\end{tabular}

. No se presupone la hipótesis nula.

b. Utilización del error estándar asintótico que presupone la hipótesis nula.

Se basa en aproximación normal. 
los que sufrieron maltrato moderado y severo mostraron una capacidad baja; encontrándose por consiguiente, a mayores niveles de maltrato físico, menores niveles de capacidad intrapersonal. Aun así, consideramos que es una relación negativa poco evidente.

A propósito de la discusión de los hallazgos con otros estudios en los que la variable maltrato físico no estuvo presente, en la dimensión intrapersonal, Cancho ${ }^{(20)}$ observó similares resultados que la del maltrato leve, pero completamente diferente en las categorías de moderado y severo, dado que los mayores porcentajes se refiere a las categorías de promedio alto y muy alto. Salirrosas (21) reportó un nivel medio, en tanto que Chávez ${ }^{(22)}$ halló una capacidad emocional y social adecuada promedio, con tendencia a una capacidad emocional y social atípica y deficiente, como lo evidencian las víctimas de maltrato físico moderado y severo, que reflejaron una capacidad emocional y social intrapersonal baja. Esto expresa, asimismo, las dificultades en la capacidad de reconocer sus propios sentimientos, dificultades en la habilidad para expresar sentimientos, creencias y pensamientos sin dañar los sentimientos de los demás, así como, defender sus propios derechos de una manera no destructiva; dificultades en la capacidad de respetarse y aceptarse a sí mismo como una persona básicamente buena o la capacidad que tienen para desarrollar sus propias capacidades potenciales que, a su vez, vería comprometida la capacidad para guiarse y controlarse a sí mismo, en su forma de pensar y actuar, y mostrarse libre de cualquier dependencia emocional.

Los niños(as) que no fueron víctimas de maltrato físico mostraron entre baja y deficiente capacidad emocional y social interpersonal. En el caso de maltrato físico leve, la capacidad encontrada fue entre muy baja y deficiente; y entre baja y deficiente capacidad interpersonal en los niños que sufrieron maltrato moderado y severo. También se encontró que, a mayores niveles de maltrato físico, menores niveles de capacidad emocional y social interpersonal; aun así, es una relación negativa poco marcada. Al contrastar con estudios que no contemplaron la variable maltrato, estos, sin embargo, muestran similitudes. Por ejemplo, Cancho ${ }^{(20)}$ evidenció en mayor porcentaje categorías en atípico deficiente, muy bajo y bajo. Herrera, Buitrago y Cepero ${ }^{(23)}$ encontraron un menor nivel de desarrollo; lo que corrobora los menores niveles de capacidad emocional y social interpersonal encontrada en víctimas de maltrato físico.

En un sentido, Salirrosas ${ }^{(21)}$ halló un nivel medio, con muy pocas diferencias hacia el nivel alto y bajo, por su parte, Chávez ${ }^{(22)}$, halló que más del 50 \% presentaron capacidad emocional y social adecuada, promedio y un mínimo porcentaje mostraron capacidad emocional y social atípica y deficiente. En esta dimensión, independientemente del nivel del maltrato físico, la capacidad emocional y social interpersonal fluctuó entre muy baja y deficiente y entre baja y deficiente, lo que indicaría las dificultades en la capacidad de los niños(as) de ser conscientes, entender y apreciar los sentimientos de los demás, asimismo, estaría también afectada la capacidad de establecer y mantener relaciones mutuamente satisfactorias, caracterizadas por la intimidad, el dar y recibir afecto y la habilidad para demostrarse a sí mismo como una persona que coopera, colabora y que es un miembro constructivo del grupo social.

Los niños(as) que no sufrieron maltrato físico mostraron una capacidad emocional y social de adaptación entre baja y deficiente; esta misma tendencia se observó en estudiantes que sufrieron maltrato físico leve, moderado y severo; no existiendo diferencias importantes entre los niveles de capacidad emocional de adaptación y los niveles de maltrato físico. Similar a estudios en los que variable maltrato no se encuentra como los resultados Chavez(22) se observó una capacidad emocional y social adecuada promedio, con tendencia marcada a una capacidad emocional y social atípica y deficiente, nivel de desarrollo marcadamente bajo, aunque diferente al de Cancho ${ }^{(20)}$ al revelar una capacidad adecuada, alta y muy alta; y Salirrosas ${ }^{(21)}$ quien evidenció un nivel medio, con tendencia a un nivel alto y bajo con pocas diferencias porcentuales.

Las víctimas de maltrato físico en las tres categorías reflejaron una capacidad emocional y social de adaptación entre baja y deficiente, por lo que se podría decir que presentan dificultades para identificar y definir los problemas como también para generar e implementar soluciones efectivas, lo que devela afectación de la habilidad para evaluar la correspondencia entre lo que experimentan subjetivamente y lo que en la realidad existe u objetivo, reflejando así dificultades en la capacidad para realizar un ajuste adecuado de sus emociones, pensamientos y conductas ante diversas situaciones y condiciones cambiantes.

Los niños(as) que no experimentaron maltrato físico, tuvieron entre adecuada y atípica excelentemente desarrollada en la capacidad emocional y social de manejo del estrés; al igual que los niños que experimentaron algún grado de maltrato físico; lo que indica que no existen diferencias importantes entre la capacidad de manejo del estrés; al maltrato físico. En un estudio similar, pero donde la variable maltrato no fue considerado, Chávez ${ }^{(22)}$ 
evidenció una capacidad emocional y social muy alta. Desarrollada. Por su parte, Salirrosas ${ }^{(21)}$ encontró un nivel medio con tendencia a un nivel alto; diferente a lo encontrado por Cancho al evidenciar resultados atípico deficiente, muy bajo y bajo. En esta dimensión de la inteligencia emocional se halló entre una adecuada y atípica excelentemente desarrollada capacidad emocional y social de manejo de estrés, por lo que los niños(as) estarían mostrando habilidad para soportar eventos adversos, situaciones estresantes, y fuertes emociones sin "desmoronarse", enfrentando activa y positivamente la tensión. Así mismo, tendrían la habilidad para resistir o postergar un impulso para actuar y controlar emociones, es decir, aceptan los impulsos agresivos, estando serenos y controlan la agresión, la hostilidad y la conducta irresponsable.

Los niños(as) que no fueron víctimas de maltrato físico o sólo en un $5 \%$, mostraron entre una baja y deficiente capacidad de estado de ánimo. Similar comportamiento se evidenció en las víctimas de maltrato físico leve, aunque en menor porcentaje, y en mayor porcentaje en víctimas de maltrato moderado y severo. Esto indica que, a menores niveles de capacidad emocional y social de estado de ánimo, mayores niveles de maltrato físico; lo es una tendencia claramente marcada, e indica una relación negativa bien marcada. Resultados similares se encuentran en estudios en los que la variable maltrato no estuvo presente. Por ejemplo, Cancho ${ }^{(20)}$ evidenció una capacidad de estado de ánimo atípico deficiente, muy bajo o bajo. Salirrosas ${ }^{(21)}$ reflejó un nivel bajo en mayor porcentaje y un nivel alto en menor porcentaje, mientras que Chávez ${ }^{(22)}$ mostró una capacidad emocional y social atípica y deficiente, con un nivel de desarrollo marcadamente bajo. Las víctimas de maltrato físico, así sea en menor o mayor porcentaje evidenciaron entre una baja y deficiente capacidad emocional y social de estado de ánimo, lo que correlaciona con dificultades en la capacidad para sentirse satisfechos con su vida, para disfrutar de sí mismos y de otros, para divertirse y expresar sentimientos positivos. De igual manera, con la capacidad de encontrar el lado más provechoso de la vida y mantener una actitud positiva, aún en la adversidad.

Los niños(as) que no sufrieron ningún tipo de maltrato físico tuvieron entre adecuada y muy alta inteligencia emocional. Los que experimentaron maltrato físico leve, moderado o severo tuvieron entre adecuada y alta. No hay diferencias importantes entre cada nivel de maltrato físico y los niveles de inteligencia emocional, no encontrándose relación importante entre inteligencia emocional y maltrato físico. En la contrastación con estudios en los que no fue considerada la variable maltrato, se encontró hallazgos similares. Por ejemplo Cancho ${ }^{(20)}$ reportó, en general, inteligencia emocional adecuada, con tendencia alta y muy alta; Manrique, un nivel excelentemente desarrollado; Chávez ${ }^{(22)}$, una capacidad emocional y social atípica, excelentemente desarrollada y capacidad emocional y social alta. Estos resultados difieren con los de Rojas ${ }^{(24)}$, que se correspondieron con una inteligencia emocional promedio, con tendencia a necesitar mejorarse, necesitar mejorarse considerablemente o marcadamente bajo; los de Alonso ${ }^{(25)}$, que reporta una inteligencia emocional adecuada, con tendencia hacia baja; y los de Salirrosas ${ }^{(21)}$, quien evidenció un nivel medio, con tendencia hacia un nivel bajo con mayor frecuencia. La inteligencia emocional entre adecuada y muy alta expresada en los resultados, en general, entre las víctimas de maltrato físico, indica que estas tienen conciencia de las sensaciones, de las emociones, expresen sus emociones, se controlan de manera que no actúan de forma desproporcionada, destructiva o inadecuada al contexto, no buscan sensaciones que generen emociones manipuladoras o mediante las cuales obtengan una ganancia secundaria ${ }^{(26)}$.

Finalmente, la inteligencia emocional encontrada fue, en general, adecuada en alta en el grupo de niñas(os) que no sufrieron ningún tipo de maltrato físico. El mismo comportamiento se evidencio en los que experimentaron algún nivel de maltrato, concluyendo que no hay relación entre los niveles del maltrato físico y los niveles de inteligencia emocional según género; sin embargo, se evidenció que las niñas alcanzaron mayores niveles en la capacidad de adaptación a comparación de los niños. Al respecto, Gutierrez ${ }^{(27)}$ reportó una puntuación final muy similar entre ambos grupos, femenino $(\bar{x}=156,19)$ y masculino $(\bar{x}=156,01)$ lo que señala que no existen diferencias en cuanto al género. Herrera, Buitrago y Cepero ${ }^{(23)}$ revelaron, sin embargo, puntuaciones más altas obtenidas por las niñas en comparación a los niños en la dimensión interpersonal, mientras que los niños obtuvieron puntuaciones más altas que las niñas en la dimensión de adaptabilidad.

\section{Conclusiones}

En relación con el objetivo general, existe relación negativa y significativa ( $r s=-0,468, p<0,05$ ) entre el maltrato físico y la inteligencia emocional, en los niños y niñas de 11 años de la I.E. $N^{\circ} 36005$ del distrito de Ascensión, Huancavelica. Además, se encuentra relación negativa baja no significativa ( $r s=-0,257$ mayor que $-0,318, p=0,119$ en niñas; $y-0,158$ mayor que -0,292, $p=0,299$ en niños) entre el maltrato físico y la inteligencia emocional, según género. La inteligencia emocional de los niños y niñas es entre adecuada y alta, sin diferencias importantes por sexo o edad. 


\section{Recomendaciones}

Actualmente, la legislación protege de la agresión física o psicológica de los directores y profesores; sin embargo, no es posible todavía restringirlos a los padres y familiares cercanos. Al igual que en los casos anteriores, urgen investigaciones cualitativas que analicen minuciosamente estos problemas, a fin de corregir la legislación peruana sobre la violencia infantil y permitir, así, que se tomen las medidas correctivas necesarias.

\section{Limitaciones}

En algunos casos de víctimas, estas tuvieron temor de contar sus experiencias de violencia familiar, lo que en algunas ocasiones dificultó la recolección de datos. En segundo lugar, teníamos que adaptarnos a la disponibilidad de ciertos docentes en ofrecernos sus horas para la recolección de los datos.

\section{REFERENCIAS}

1. Bar-On, R. The Emotional Quotient inventory (EQ-I): Techical Manual, Toronto: Multi-Health Systems. 1997.

2. Soriano A. Maltrato Infantil. Madrid: San Pablo, 2001.

3. Ministerio de la Mujer y Poblaciones Vulnerables. Programa Nacional Contra la Violencia Familiar y Sexual. Sistema de Registros de Casos y Atenciones en los Centros de Emergencia. Lima; 2016, 2017 y 2018.

4. Encuesta Nacional Sobre Relaciones Sociales (ENARES). Lima; 2016.

5. Sarrionandia A, Garaigordobil M. Programas de Inteligencia Emocional: Una revisión. En: VIII Congreso Internacional de Psicología y Educación. Alicante: (Editorial); 2016.

6. Boj J. Odontopediatría. Masson-España; 2005

7. Trigoso L. Tipos de maltrato infantil que presentan los estudiantes de la Institución Educativa Primaria Pedro Castro Alva N 18006 [Tesis de grado en Enfermería] Chachapoyas: Universidad Nacional Toribio Rodríguez de Mendoza de Amazonas; 2017.

8. Salcedo DM. Maltrato infantil y sus características socioculturales en escolares de la Institución Educativa No 10381. Chota [Tesis de maestría en Ciencias] Cajamarca: Universidad Nacional de Cajamarca; 2018.

9. Huamán M. Eficacia del programa educativo en el aprendizaje de habilidades para la vida en víctimas de maltrato infantil en la Institución Educativa № 36009 del Distrito de Huancavelica [Tesis doctoral ]. Huancavelica: Universidad Nacional de Huancavelica; 2011.

10. Carhuachuco. E. Maltrato Infantil y Relacionado con la Conducta Asertiva en los niños/niñas de la Institución Educativa Ramón Castilla y Marquesado [Tesis de doctoral]. Huancavelica: Universidad Nacional de Huancavelica; 2010.

11. Belito Mancha E, Gaspar Aparco L. Maltrato infantil y nivel de asertividad en los estudiantes de la Institución Educativa, José María Arguedas. Lircay [Tesis de pre grado]. Huancavelica: Universidad Nacional de Huancavelica; 2009.

12. Castrejón A. El maltrato y los derechos del niño en la Localidad de Huancavelica. [Tesis de maestría en Salud Pública]. Huancavelica: Universidad Nacional de Huancavelica. 2009.
13. Uculmana C, Lanchipa A. Como hacer tesis y trabajos de investigación. Lima, 2000.

14. Ugarriza N, Pajares L. La evaluación de la inteligencia emocional a través del inventario de BarOn (I-CE) en una muestra de Lima metropolitana. Lima; 2001.

15. Peña, K. Nivel de maltrato infantil intrafamiliar en niños de 5 to y 6 to de primaria en una institución educativa. Cercado de Lima. 2016. [Tesis de grado en enfermería]. Lima: Universidad Nacional Mayor de San Marcos; 2017.

16. Bautista R. Maltrato infantil en niños (as) de 6 grado de primaria en la institución educativa "Juan Andrés Vivanco Amorin" en el distrito de Ate Vitarte, periodo 2018. [Tesis de grado en Psicología] Lima: Universidad Nacional Inca Garcilaso de la Vega; 2019.

17. Saucedo R. El maltrato infantil y el rendimiento académico de los estudiantes de la Institución Educativa $\mathrm{N}^{\circ}$ 821010 San Isidro - José Sabogal -San Marcos 2014. [Tesis de maestría en Enfermería] Universidad Nacional de Cajamarca. Cajamarca. Perú 2019

18. Huaman A. Maltrato infantil y estado de ánimo en estudiantes de la Institución Educativa Ramón Castilla y Marquesado- Localidad de Huancavelica. Universidad Nacional de Huancavelica. Huancavelica. Perú. 2014.

19. Belito M y Gaspar A. Maltrato infantil y nivel de asertividad en los alumnos de la Institución Educativa, José María Arguedas. Lircay. [Tesis de grado en Educación]. Huancavelica. 2009.

20. Cancho K. Clima social familiar y relación con inteligencia emocional en estudiantes del sexto grado de primaria de las instituciones educativas gestión pública distrito de Cerro Colorado 2018 [Tesis de maestría en Ciencias,con mención en Psicología Clínica-Educativa, Infantil y Adolescencial] Arequipa: Universidad Nacional de San Agustín de Arequipa; 2018.

21. Salirrosas J. Inteligencia emocional y aptitudes perceptivas de atención en estudiantes de cuarto y quinto grado de primaria de una Institución Educativa [Tesis de maestría en Problemas de aprendizaje] Universidad Cesar Vallejo; 2017.

22. Chávez NV. Inteligencia emocional en niños de quinto grado de primaria de la I.E. Tnt. Crl. Leopoldo Pérez Salmon de Carmen de la Legua - Callao, 2018 [Tesis de grado en Psicología] Lima: Universidad Inca Garcilaso de la Vega; 2018.

23. Herrera L, Buitrago R y Cepero S. Emotional Intelligence in Colombian Primary School Children. Location and Gender. Analysis. Universitas Psychologica, 2017. 16(3), 1-10. Colombia.

24. Rojas E. Inteligencia emocional y el nivel de autoestima en niños de 9, 10 y 11 años de la Institución Educativa Vista Alegre del distrito de Puente Piedra [Tesis de maestría en Ciencias de La Educación] Lima: Universidad Nacional de Educación Enrique Guzmán y Valle; 2018.

25 . Alonso $D$. La relación entre inteligencia emocional y el rendimiento académico en el área de Matemática en niños de tercer grado de primaria de la institución educativa Bertolt Brecht de la provincia de Barranca en el año 2013 [Tesis de maestría en Psicología Educativa] Lima: Universidad Cesar Vallejo; 2018

26. Ramos N, Recondo O, Enríquez H. Practica la inteligencia emocional plena. Barcelona: Kairos; 2013.

27. Gutiérrez MA. La inteligencia emocional en la escuela. Valoración de la inteligencia emocional de los profesores y estudiantes [Trabajo fin de grado en Ciencias de la Educación] Sevilla: Universidad de Sevilla; 2017. 